\title{
Carcinoembryonic antigen-targeted photodynamic therapy in colorectal cancer models
}

\author{
Fortuné M. K. Elekonawo ${ }^{1 *}$, Desirée L. Bos ${ }^{1}$, David M. Goldenberg ${ }^{2,3}$, Otto C. Boerman ${ }^{1}$ and Mark Rijpkema ${ }^{1}$
}

\begin{abstract}
Background: In colorectal cancer, survival of patients is drastically reduced when complete resection is hampered by involvement of critical structures. Targeted photodynamic therapy (tPDT) is a local and targeted therapy which could play a role in eradicating residual tumor cells after incomplete resection. Since carcinoembryonic antigen (CEA; CEACAM5) is abundantly overexpressed in colorectal cancer, it is a potential target for tPDT of colorectal cancer.

Methods: To address the potential of CEA-targeted PDT, we compared colorectal cancer cell lines with different CEA-expression levels (SW-48, SW-480, SW-620, SW-1222, WiDr, HT-29, DLD-1, LS174T, and LoVo) under identical experimental conditions. We evaluated the susceptibility to tPDT by varying radiant exposure and concentration of our antibody conjugate (DTPA-hMN-14-IRDye700DX). Finally, we assessed the efficacy of tPDT in vivo in 18 mice (BALB/cAnNRj-Foxn 1 ${ }^{\text {nu/nu}}$ ) with subcutaneously xenografted LoVo tumors.
\end{abstract}

Results: In vitro, the treatment effect of tPDT varied per cell line and was dependent on both radiant exposure and antibody concentration. Under standardized conditions $\left(94.5 \mathrm{~J} / \mathrm{cm}^{2}\right.$ and $0.5 \mu \mathrm{g} / \mu \mathrm{L}$ antibody conjugate concentration), the effect of tPDT was higher in cells with higher CEA availability: SW-1222, LS174T, LoVo, and SW$48(22.8 \%, 52.8 \%, 49.9 \%$, and $51.9 \%$ reduction of viable cells, respectively) compared to cells with lower CEA availability. Compared to control groups (light or antibody conjugate only), tumor growth rate was reduced in mice with s.c. LoVo tumors receiving tPDT.

Conclusion: Our findings suggest cells (and tumors) have different levels of susceptibility for tPDT even though they all express CEA. Furthermore, tPDT can effectively reduce tumor growth in vivo.

Keywords: Colorectal cancer, Targeted photodynamic therapy, Carcinoembryonic antigen, Targeted, IRDye700DX

\section{Introduction}

Surgery is a cornerstone in curative treatment of colorectal cancer; however, incomplete resection can drastically reduce survival after surgery $[1,2]$. Radical resection of tumors might be hampered by involvement of critical structures including large vessels, nerves, or visceral organs. In advanced-stage cancers, adjuvant local or systemic therapies can improve survival after (incomplete) resection. Survival of systemically treated patients with advanced colorectal cancer has improved with modern

\footnotetext{
* Correspondence: fortune.elekonawo@radboudumc.nl

'Department of Radiology and Nuclear Medicine, Radboud University

Medical Center, PO Box 9101, 6500 HB Nijmegen, The Netherlands

Full list of author information is available at the end of the article
}

systemic therapies [3], but the maximum dose of systemic therapy is limited by toxicity and side effects [4].

Photodynamic therapy (PDT) could play a role in overcoming the limitations of incomplete radical resection and toxicity from systemic therapy. PDT has gained a role in treatment in different fields of oncology nowadays [5-8]. It can be applied as standalone treatment modality, although it might also serve as adjuvant treatment to surgery after incomplete resection [9].

The principle of PDT is based on combining three non-toxic components: oxygen, light, and a photosensitizer. The photosensitizer is excited with the physical energy of non-ionizing light (of a specific wavelength) which, through a series of photochemical reactions, 
results in formation of highly reactive oxygen species (ROS) $[10,11]$. In turn, ROS may induce local cell apoptosis and necrosis and/or cause microvascular damage. Furthermore, a change in photosensitizer structure and hydrophilicity has been proposed to contribute to cell damage [12]. Preferably, accumulation of the photosensitizer should be tumor-specific to prevent extensive damage to the normal tissue surrounding the tumor and to increase the intratumoral dose. Therefore, a tumortargeted PDT approach has been developed [13]. In targeted photodynamic therapy (tPDT), a tissue of interest is selectively localized using a targeting vehicle conjugated to a photosensitizer. When the photosensitizervehicle conjugate has accumulated in targeted tissue, light of a specific wavelength is administered locally, making this therapy highly specific.

Carcinoembryonic antigen (CEA) is a membraneanchored glycoprotein and is overexpressed in 90-95\% of colorectal cancer cases. Therefore, CEA can be used for primary targeting of colorectal carcinomas. hMN-14 (labetuzumab) is an IgG directed against the carcinoembryonic antigen-related cell adhesion molecule 5 with high affinity [14]. However, different tumors express different amounts of CEA. Therefore, in clinical practice CEA-targeted PDT might only be useful in tumors with sufficient CEA expression, when complete tumor resection is hampered by the presence of critical structures that are to be preserved.

Here, we investigate whether the effect of tumortargeted PDT is influenced by the availability of CEA on the cell surface of tumor cells with different CEA expression levels. Our multimodal conjugate (DTPA-hMN-14700DX) consists of the humanized anti-CEA antibody, hMN-14 (labetuzumab), the photosensitizer IRDye700DX, and the chelator diethylenetriaminepentaacetic acid (DTPA). DTPA allows radiolabeling with ${ }^{111}$ In and subsequent in vivo tumor detection and precise quantification of the antibody conjugate accumulation. After the in vitro experiments on different colorectal cancer cell lines, the therapeutic effect of tPDT was evaluated in vivo in a xenograft mouse model.

\section{Materials and methods}

\section{Cell culture}

CEA-expressing human colon adenocarcinoma (primary or metastatic) cell lines were obtained from the American Type Culture Collection (ATCC, Manassas, VA, USA). SW-1222 cells were obtained from SigmaAldrich (Saint Louis, MO, USA). LS174T, SW-620, SW-480, SW-48, DLD-1, and HT-29 were cultured in RPMI-1640 (Gibco ${ }^{\mathrm{TM}}$, Dun Laoghaire, Ireland) supplemented with $10 \%$ fetal bovine serum (FBS) and 2 mML-glutamine. WiDr and SW-1222 were cultured in DMEM high glucose (Gibco) supplemented with
$10 \%$ FBS. LoVo was cultured in Ham's F-12 Nutrient mix GLUTAMAX (Gibco) supplemented with $10 \%$ FBS. No antibiotic additives were used. All cells tested negative for Mycoplasma. Cells were cultured in tissue culture flasks in a humidified incubator at $37{ }^{\circ} \mathrm{C}$ in an atmosphere of $95 \%$ air and $5 \% \mathrm{CO}_{2}$. Cells were harvested with $0.05 \%$ trypsin-EDTA (ethylenediaminetetraacetic acid).

\section{DTPA-hMN-14-IRDye700DX conjugation}

hMN-14 (labetuzumab) was kindly provided by Immunomedics, Inc., Morris Plains, NJ, USA. It was conjugated with IRDye700DX-NHS (LI-COR, Lincoln, NE, USA) and SCN-Bz-dieethylenetriaminepentaacetic acid (DTPA) (Macrocyclics, Plano, TX, USA) in two steps. First, hMN-14 was conjugated with IRDye700DX-NHS in $0.1 \mathrm{M} \mathrm{NaHCO}, \mathrm{pH} 8.5$, with a 10 -fold molar excess of IRDye700DX-NHS. Next, the reaction mixture was incubated for $1 \mathrm{~h}$ at room temperature on an orbital shaker and protected from light. Second, SCN-Bz-DTPA in $0.1 \mathrm{M} \mathrm{NaHCO}, \mathrm{pH} 9.5$ was added to the reaction mixture in a 10-fold molar excess. After another hour of incubation on the orbital shaker in the dark, the mixture was dialyzed in a Slide-A-Lyzer ( $10 \mathrm{kDa}$ cutoff; Thermo Fisher Scientific, Waltham, MA, USA) against phosphate-buffered saline (PBS) containing 2 g/L Chelex 100 Resin (Bio-Rad Laboratories, Inc.; Hercules, CA, USA). The final concentration of the conjugate was determined spectrophotometrically at $280 \mathrm{~nm}$ (Ultrospec 2000 spectrophotometer; Pharmacia Biotech), correcting for the absorption of IRDye700DX at that wavelength (3\%, according to the manufacturer's protocol). The molar substitution ratio of IRDye700DX was determined spectrophotometrically at $648 \mathrm{~nm}$ and reached 4.5 .

\section{Radiolabeling of the hMN-14 conjugate}

Briefly, $\left.{ }^{[111} \mathrm{In}\right] \mathrm{InCl}_{3}$ (Curium, Petten, The Netherlands) was added to DTPA-hMN-14-IRDye700DX in $3 \mathrm{~V}$ of 0.1 M 2-(N-morpholino)ethanesulfonic acid (MES), $\mathrm{pH}$ 5.5. After $30 \mathrm{~min}$ of incubation at room temperature, 50 $\mathrm{mM}$ EDTA was added to the labeling reaction to a final concentration of $5 \mathrm{mM}$ to chelate unincorporated $\left[{ }^{111} \mathrm{In}\right] \mathrm{InCl}_{3}$. Labeling efficiency was determined by instant thin-layer chromatography on Varian silicagel strips (ITLC-SG; Agilent Technologies, Amstelveen, The Netherlands) using $0.1 \mathrm{mM}$ ammonium acetate $\left(\mathrm{NH}_{4} \mathrm{Ac}\right)$ buffer with 0.1 M EDTA, pH 5.5 as the mobile phase and labeling efficiency reached $>95 \%$.

For the in vitro binding assay, DTPA-hMN-14IRDye700DX was radiolabeled with $0.5 \mathrm{MBq} / \mu \mathrm{g}$ of $\left[{ }^{111} \mathrm{In}\right] \mathrm{InCl}_{3}$. For the biodistribution studies, in two mice with s.c. LoVo tumors, DTPA-hMN-14-IRDye700DX was radiolabeled with $0.23 \mathrm{MBq} / \mu \mathrm{g}$ of $\left[{ }^{111} \mathrm{In}\right] \mathrm{InCl}_{3}$. 


\section{In vitro binding assay}

The radiolabeled conjugate was diluted to contain 2.4 $\mathrm{kBq}$ per $\mu \mathrm{L}$. To count the amount of activity, we used a shielded 3"-well-type $\gamma$-counter (Perkin-Elmer, Boston, MA, USA). Cells were counted and placed in culture medium supplemented with 0.5\% Bovine Serum Albumin (BSA; Sigma-Aldrich Chemie N.V., Zwijndrecht, Netherlands) (Binding buffer; $\mathrm{BB}$ ) at a concentration of $1 \times 10^{7}$ cells per milligram. Next, $4 \times 10^{6}$ cells were pipetted in a $1.5 \mathrm{~mL}$ eppendorf tube ( 2 tubes per cell line; experiment performed in triplicate). We added $240 \mathrm{kBq}$ of our radiolabeled antibody conjugate to cells in each tube. An excess of unlabeled DTPA-hMN-14IRDye700DX $(1.9 \mu \mathrm{g})$ was added to the last three tubes of each cell lines to determine the amount of nonspecific binding of the conjugate.

Cells were incubated at $37^{\circ} \mathrm{C}$ with $5 \% \mathrm{CO}_{2}$ for $4 \mathrm{~h}$. Following incubation, cells were centrifuged at $805 \mathrm{~g}$ for $5 \mathrm{~min}$, the supernatant was removed and the remaining activity in the tubes was measured in a $\gamma$-counter. A $100-\mu \mathrm{L}$ standard, representing $100 \%$ activity, was measured in triplicate simultaneously. The antibody binding in the presence of an excess unlabeled antibody was subtracted from the antibody conjugate binding for each cell line. This results in a measure for the specific binding of our antibody conjugate, expressed as percentage of the total amount of added antibody conjugate.

\section{PDT device}

We used a standardized $690 \mathrm{~nm}$ (SMBB690D-1100-02) high-output LED (Marubeni America Corporation; Santa Clara, CA, USA) device for illumination of targets [15]. All experiments were performed with the device set to its maximum power output of $200 \mathrm{~mW} / \mathrm{cm}^{2}$. To adjust light fluency rate, we varied the distance between light source and target. The bottom of the in vitro setup was transparent to prevent excessive heat generation and light reflection from the surface beneath the treated cells.

\section{In vitro photodynamic therapy}

To gain insight into the effects of tPDT and CEA availability, LoVo, LS174T, DLD-1, and HT-29 cells were treated with different antibody conjugate doses and light doses (Fig. 1). Next, to directly compare the therapeutic effect on the 9 cell lines, all cells were treated with the same light and antibody-conjugate dose. Briefly, $2 \times 10^{5}$ cells per well were plated in 2 wells of a 24-well plate
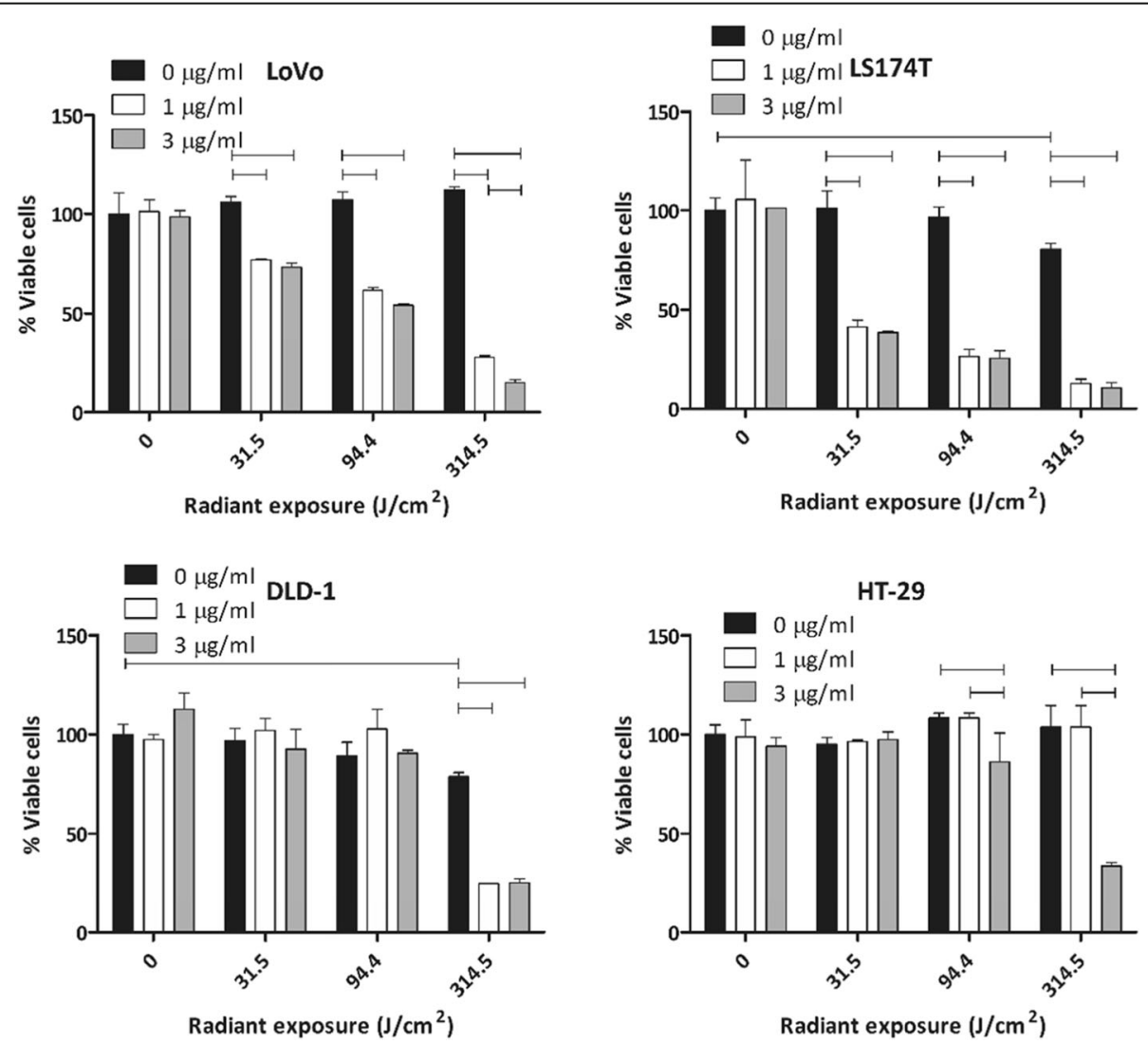

Fig. 1 In vitro cell viability (tPDT effect) on LoVo, LS174T, DLD-1, and HT-29 cells treated with different doses of light and antibody conjugate concentration. Statistically significant differences $(p<0.05)$ are indicated with horizontal bars 
(experiment performed in triplicate) 1 day prior to treatment. On the day of treatment, $0.5 \mu \mathrm{g} / \mu \mathrm{L}$ DTPA-hMN14-700DX was added in $500 \mu \mathrm{L}$ of BB. In this experiment, tPDT was performed with a radiant exposure of $94.5 \mathrm{~J} / \mathrm{cm}^{2}$.

For both in vitro experiments, after harvesting, cells were plated in transparent 24-well plates (Corning Inc.; Corning, NY, USA) $\left(1.5-2.0 \cdot 10^{5}\right.$ cells per well) and allowed to adhere overnight. After cells were adherent, they were incubated with the antibody-conjugate in $\mathrm{BB}$ for $4 \mathrm{~h}$. Following incubation, BB was removed and cells were washed once with phosphate-buffered saline to remove unbound antibody. Next, fresh medium was added and cells were exposed to specific radiant exposures. Cells rested for $1 \mathrm{~h}$ after light exposure. Next, cells were washed to remove cell debris. Hereafter, cell viability was assessed using the cell titer $\mathrm{Glo}^{\circ}$ (Promega Corporation; Madison, WI, USA) luminescent cell viability assay. Incubation in demineralized water was used as positive control for $100 \%$ cell death.

\section{Animals}

All animal experiments were approved by the Dutch Central Committee for Animal Experiments, and local protocols were approved by the Institutional Animal Welfare Committee of the Radboud University Medical Center and were conducted in accordance to the guidelines of the Revised Dutch Act on Animal Experimentation (2014).

Twenty male BALB/cAnNRj-Foxn $1^{n u / n u}$ nude mice (7 to 9 weeks old, $18-22 \mathrm{~g}$ body weight; Janvier labs; Le Genest-Saint-Isle, France) were housed in individually ventilated cages (5 mice per cage) under standard nonsterile conditions. Mice had free access to standard animal chow and water. Animals were adapted to laboratory conditions for 1 week before experimental use. Subcutaneous (s.c.) tumors were induced by s.c. injection of $5 \times 10^{6}$ freshly harvested LoVo cells. Tumors grew in all mice that were injected s.c.

\section{In vivo targeted photodynamic therapy}

When average tumor size reached $45 \mathrm{~mm}^{3}$ and after stratification based on tumor size, 18 mice were randomly allocated into three experimental groups (treatment $\mathrm{tPDT}, \mathrm{PBS}+0.5 \%$ BSA with light exposure, antibody-conjugate without light exposure), 6 mice per group), mice were injected with $30 \mu \mathrm{g}$ of unlabeled DTPA-hMN-14-IRDye700DX or PBS + 0.5\% BSA via a $200-\mu l$ tail vein injection.

Tumors of mice in one control group $(n=6)$ and the treatment group $(n=6)$ were selectively exposed to 300 $\mathrm{J} / \mathrm{cm}^{2}$ of near infrared (NIR) light under inhalation anesthesia $\left(2.5 \%\right.$ isoflurane mixed with $100 \% \mathrm{O}_{2}(1 \mathrm{~L} /$ $\mathrm{min})$ ). All mice, including non-irradiated controls, were anesthetized for $12 \mathrm{~min}$. The liver and other organs were protected from exposure to the NIR light by covering those areas with a gauze and aluminum foil.

Treatment efficacy was determined based on tumor growth. Tumor diameters were measured in three dimensions by a blinded observer using a caliper three times per week. Tumor volume was calculated as the volume of an ellipsoid: $4 / 3 \cdot \pi \cdot r 1 \cdot r 2 \cdot r 3$. Herein, $r$ was calculated by dividing the tumor length, width, or height by two. Mice were euthanized by $\mathrm{O}_{2} / \mathrm{CO}_{2}$ asphyxiation when tumor volume exceeded more than $1000 \mathrm{~mm}^{3}$. One mouse in the treatment group was excluded from the analyses, since we failed to irradiate its tumor with light (the aluminum foil shifted and covered the tumor).

\section{Biodistribution}

Two mice were used to determine the biodistribution of ${ }^{111}$ In-labeled DTPA-hMN-14-IRDye700DX (Additional file 1: Figure S2) ex vivo. Twenty-four hours after injection of the tracer, mice were euthanized and tissues of interest (tumor, muscle, lung, spleen, kidney, liver, pancreas, stomach, and duodenum) were dissected and weighed after which activity was measured in the $\gamma$ counter. Blood samples were obtained by cardiac puncture. For calculation of the uptake of activity in each tissue as a fraction of the injected activity, three aliquots of the injection dose were counted in the $\gamma$-counter simultaneously.

\section{Statistical analyses}

Statistical analyses were performed using GraphPad Prism version 5.03 (GraphPad Software, Inc.; San Diego, CA, USA) and Statistical Package for Social Sciences, Version 22.0 (IBM Corp.; Armonk, NY, USA). A twoway ANOVA with Bonferroni correction for multiple testing was performed to analyze the effects of radiant exposure and antibody dose in the in vitro experiment with 9 different colorectal cancer cell lines. Furthermore, a one-way ANOVA with Dunnet correction for multiple testing was performed to compare the control condition without antibody and light (absolute control) with the control condition with light (intern control). For the in vivo experiment, a one-way ANOVA with Bonferroni correction for multiple testing was performed. A $p$ value $<0.05$ was used to reject the null hypothesis. Data are presented as mean and standard deviation.

\section{Results}

First, we assessed the binding capacity of ${ }^{111}$ In-DTPAhMN-14-IRdye700DX to cell lines with different CEA expression levels. This capacity can be considered as a surrogate value for accessible CEA epitopes on the cell surface. Additional file 2: Figure S1 illustrates that the 
cells used in this study can be roughly classified into two groups: low and high CEA accessibility.

To gain insight into the effects of tPDT, we treated 2 cell lines with low CEA availability and 2 cell lines with high CEA availability under different conditions, varying both the antibody conjugate concentration and radiant exposure (light dose).

LS174T and LoVo cells (both high CEA availability) showed a light dose-dependent increase of therapeutic efficacy of tPDT, regardless of antibody conjugate concentration (Fig. 1). At the highest light dose $(314.5 \mathrm{~J} /$ $\mathrm{cm}^{2}$ ), the effect of tPDT on LoVo was higher at $3 \mu \mathrm{g} / \mathrm{mL}$ compared to $1 \mu \mathrm{g} / \mathrm{mL}$ antibody conjugate concentration ( $27.8 \%$ vs $15.1 \%$ viable cells, $p=0.002)$. At lower light doses, we did not observe an additional effect of increasing the antibody conjugate dose. Similar to LoVo, in LS174T the effect of tPDT increased with increasing light dose (Fig. 1). So, in the cell lines with higher CEA availability, we observed a clear effect of increasing light dose and antibody conjugate dose on the effect of tPDT. In contrast, in cell lines with lower CEA availability (DLD-1 and HT-29), we did not observe this variation in susceptibility for tPDT. Decreased cell viability due to tPDT in DLD-1 was only observed at the highest dose level of light $(p<0.001)$ and was not dependent on the antibody conjugate concentration used. In HT-29 cells (low to moderate CEA availability), we did not find an effect of tPDT at $1 \mu \mathrm{g} / \mathrm{mL}$ of antibody conjugate. At $3 \mu \mathrm{g} / \mathrm{mL}$, however, there was an effect of tPDT at the two highest light doses $\left(94.4\right.$ and $\left.314.5 \mathrm{~J} / \mathrm{cm}^{2}\right)$. From these experiments, we concluded that the effect of antibody conjugate dose and light dose on the efficacy of tPDT seem to be dependent on the CEA availability in tumor cells.
Subsequently, we performed a separate experiment to investigate the relationship between CEA availability and tPDT effects. To explore this relationship, we included 9 cell lines with varying CEA expression levels. Figure 2 summarizes our findings on tPDT effect and its relation to amount of antibody conjugate binding of the different cell lines. No treatment effect was observed in cells with less than $5 \%$ specific binding of the antibody conjugate, which represents low CEA availability (HT-29, SW620, SW480, WiDr, and DLD-1). SW-1222 (5.5\% specific binding) showed a moderate effect of tPDT after treatment (78.2\% viable cells). LS174T, LoVo, and SW48 (7.7, 11.7 , and $30.6 \%$ specific binding, respectively) were more sensitive to treatment with tPDT $(47.2 \%, 50.1 \%$, and $48.1 \%$ viable cells after treatment, respectively), suggesting that a minimum level of antibody conjugate binding to the cells is required for effective treatment with tPDT.

Subsequently, we evaluated the ability of tPDT to reduce in vivo tumor progression in a xenograft mouse model. Before evaluating the treatment, we performed an ex vivo biodistribution of ${ }^{111} \mathrm{In}$-labeled DTPA-hMN-14-IRDye700DX. We found $13.7 \pm 3.8$ $\% \mathrm{ID} / \mathrm{g}$ of our tracer in tumor, $8.8 \pm 0.5 \% \mathrm{ID} / \mathrm{g}$ in spleen and $29.4 \pm 2.6 \% \mathrm{ID} / \mathrm{g}$ in liver tissue (Additional file 2: Figure S1). In mice treated with tPDT, we generally observed slower tumor growth than the control groups $(n=17, p>0.05)$. The tumor of one mouse in the treatment group, however, showed a more aggressive growth pattern after treatment (mouse 1, Fig. 3). This was possibly caused by incomplete tumor irradiation during tPDT, but because this was not reported during treatment of this mouse, this dataset was not rejected from the analyses.

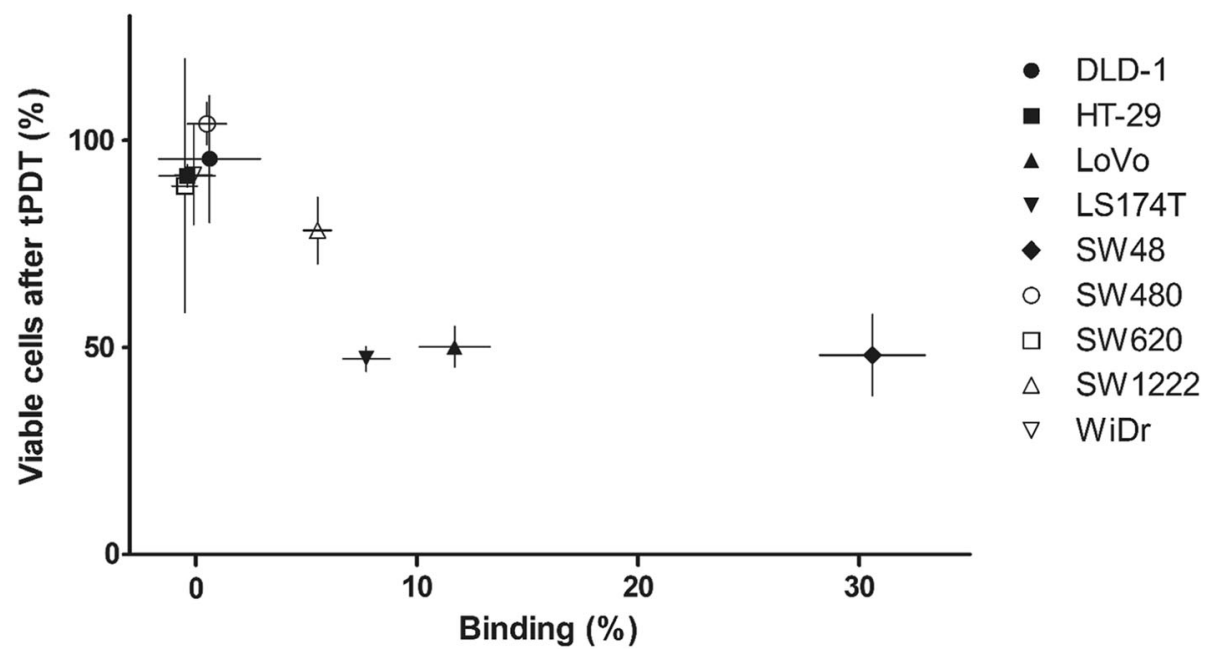

Fig. 2 Relation between the tPDT effect (amount of remaining viable cells $1 \mathrm{~h}$ after treatment) and antibody conjugate binding (expressed as percentage of the total amount of added antibody conjugate) for the nine different cell lines. Note that in general more binding (more accessible/targetable CEA sites) leads to a greater effect of tPDT (fewer viable cells) 


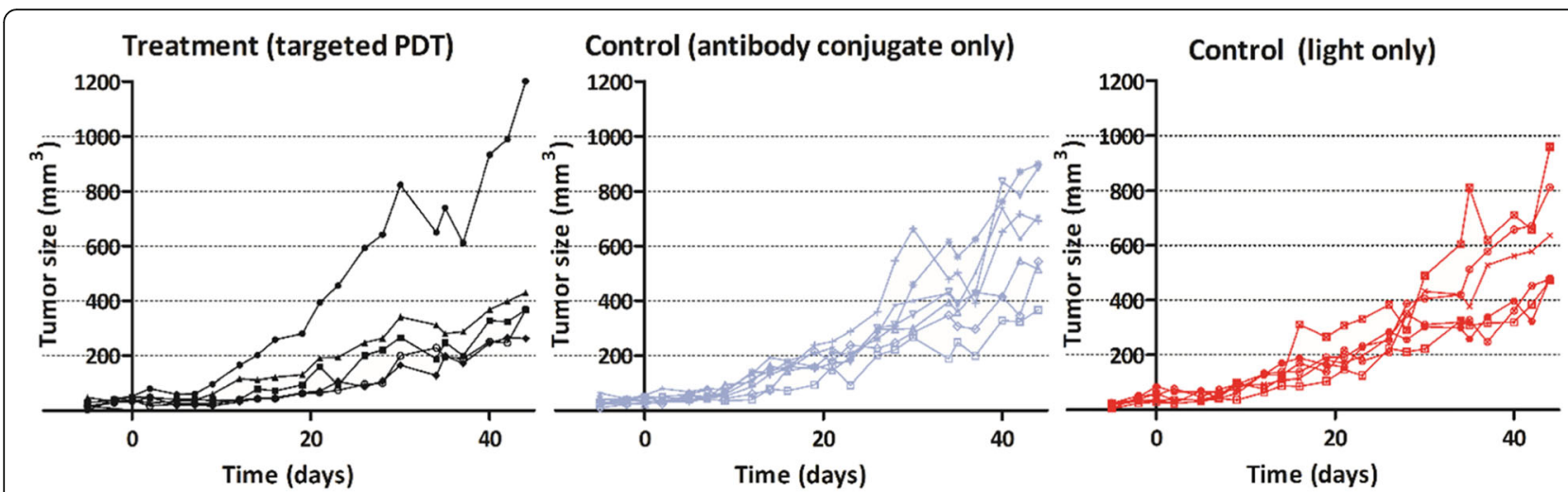

Fig. 3 In vivo tumor growth progression after targeted PDT treatment (day 0 ) and control conditions followed up three times per week. The two control groups: DTPA-hMN-14-IRDye700DX only group (without light exposure) in blue and the light-only group in red. Only 5 mice were treated due to a technical incident during the treatment of the 6th mouse. In general, mice in the tPDT (treatment) group show a delayed tumor growth pattern. One of the treated mice had a more aggressive growth pattern

\section{Discussion}

The current study demonstrates that in vitro tumor cell lines with different levels of CEA targetability have varying susceptibility to tPDT. Furthermore, the amount of ${ }^{111}$ In-DTPA-hMN-14-IRDye700DX binding to these cells seems to play a crucial role in the efficacy of tPDT. Our findings suggest that only cell lines that can be targeted with higher amounts of antibody-conjugate can be treated effectively with tPDT. In vivo, we observed a trend towards tumor growth delay in xenografted tumors of tPDT-treated mice.

In the tested cell lines, higher light dose increased the efficacy of tPDT. However, in DLD-1 and LS174T cells, the maximum light dose led to a statistically significant decrease in cell viability even without the presence of the antibody conjugate (Fig. 1). This suggests that the energy provided by the light source may have been excessive in this condition. Hyperthermia can lead to decreased cell survival in (monolayer) cells and is likely to be responsible for the decrease in cell viability of the $0 \mu \mathrm{g} / \mathrm{mL}$ control condition of LS174T and DLD-1 $[16,17]$.

In this study, we included a large series of CEAexpressing cell lines in a direct comparison. To compare the tPDT effect in all cell lines under identical conditions, we performed a single experiment with fixed conditions: $\quad 0.5 \mu \mathrm{g} / \mu \mathrm{L} \quad{ }^{111}$ In-DTPA-hMN-14-IRDye700DX and total radiant exposure $94.5 \mathrm{~J} / \mathrm{cm}^{2}$. Higher radiant exposures could have an additional thermal effect on several cell lines (see above). Since we chose $94.5 \mathrm{~J} / \mathrm{cm}^{2}$, the observed effects depicted in Fig. 2 are considered to be only tPDT-mediated, but may be an underestimation of the maximum tPDT effect that could be achieved. More specific binding of ${ }^{111}$ In-DTPA-hMN-14-IRDye700DX resulted in a larger treatment efficacy of tPDT in vitro (Fig. 2). These findings are consistent with previous studies that showed an increased efficacy of tPDT when there is more photosensitizer available in the tumor [13].

Increasing the number of photosensitizer moieties per antibody molecule (substitution ratio) might further increase the amount of photosensitizer in tumors. However, as we use a random conjugation method, excessive conjugation of the photosensitizer to an antibody might affect the antibody binding affinity. Additionally, when conjugating high amounts of a photosensitizer or in general a fluorescent dye to an antibody, changes in chemical properties (lipophilicity and net charge) may lead to faster blood clearance, less tumor uptake, and more accumulation in liver and spleen [18-20]. Findings from these studies are in line with the findings of the biodistribution performed in the current study. Random conjugation strategies can, therefore, be considered a limitation regarding the preparation of an antibodyphotosensitizer conjugate. Our results also suggest that tPDT efficacy is dependent on a combination of radiant exposure and amount of photosensitizer that is present on the target. In addition, different coping strategies of tumor cells to tPDT-induced damage may affect treatment efficacy, but this was not taken into account in the current study.

In contrast to in vitro tPDT, in vivo tPDT can be particularly challenging due to the multifactorial nature of the treatment and treatment effect. In the current study, we tested the efficacy of tPDT with subcutaneous LoVo tumors in BALB/c nude mice. LoVo cells were chosen as a representative because the antibody-conjugate binding to the cells was the most representative of the 3 cell lines with the a highest binding percentage (SW-48, LoVo, and LS174T) (Additional file 2: Figure S1). We observed a steady increase in tumor size in the control groups during 6 weeks. The treated mice showed a slower tumor growth pattern in general (Fig. 3). The 
delay in tumor growth was, however, not statistically different compared to the control groups. This was caused by one treated mouse that showed an accelerated tumor growth pattern. When cells survive after (incomplete) tPDT, cell cycle progression might be stimulated, resulting in increased proliferation, and invasive and metastatic growth [21]. This can particularly occur when treatment is not adequate. Furthermore, in a single tumor, heterogeneity in CEA expression may exist, which could be a limitation in adequate tumor targeting [22, 23]. Earlier studies indicate that host immunity can also play a crucial role in achieving a successful effect of (targeted) PDT [10, 24].

However, due to the use of an immunodeficient mouse model, the effects of immune system involvement could not be assessed in the current study. Moreover, fractionated and repeated tPDT might enhance the tPDT effect, as observed earlier by Mitsunaga and colleagues [25].

During the past years, targeted PDT approaches have gained more clinical and scientific interest. In the clinical situation, tPDT may mainly have a role as adjuvant therapy to surgery [9]. The main advantage of tPDT over other adjuvant therapies is the ability to selectively apply therapy to local areas and tissues of interest, thereby minimizing side effects. When combined with NIR fluorescence or nuclear imaging, its potential for clinical use as theranostic approach is highlighted even further [26, 27].

Here, we focused on tPDT in a colorectal cancer model using a humanized monoclonal antibody directed against CEA (CEACAM5). Since other primary epithelial malignancies also express varying levels of CEA, CEAtargeted PDT could potentially be used in treatment of other malignancies as well. These include carcinomas of the gall bladder, urinary bladder, stomach, pancreas, ovary, endometrium, and lung [28]. Other conjugates of the same antibody have already been used in (clinical) studies investigating fluorescence and radioguided surgery, radioimmunotherapy, and antibody-drug conjugates [29-35].

Our findings are in line with work by Shirasu et al., who showed phototoxic and dose-dependent effects of CEA-targeted PDT, with their anti-CEA antibody C2-45 conjugated with IRDye700DX [36]. Our radiolabeled antibody conjugate, ${ }^{111}$ In-DTPAhMN-14-IRDye700DX, allows precise quantification of the tracer both in vivo and in vitro, and may enable SPECT or PET imaging prior to surgery. During surgery, when tumors are covered by overlying tissue, the radiolabel could guide the surgeon to the area of interest, using a gamma probe prior to resection of tumors [29, 37]. Subsequently, tumor margins may be visualized using near-infrared fluorescence imaging using the same radiolabeled antibody conjugate. Lastly, the photosensitizer can be irradiated to treat residual tumor that was excluded from excision [9].

\section{Conclusions}

Our findings suggest cells (and tumors) have different levels of susceptibility for tPDT even though they all express CEA. Furthermore, tPDT can effectively reduce tumor growth in vivo. In the current study, we treated whole subcutaneous xenografted tumors, whereas in the clinical situation one would optimally use tPDT after resection to treat (microscopically) small amounts of residual tumor cells. Thus, our findings could even be an underestimation of clinically achievable results with tPDT.

\section{Supplementary information}

Supplementary information accompanies this paper at https://doi.org/10. 1186/s13550-019-0580-z.

Additional file 1: Figure S2. Ex vivo biodistribution of two mice with subcutaneous LoVo tumors, one day after ${ }^{111}$ In-labeled DTPA-hMN-14IRDye700DX injection.

Additional file 2: Figure S1. the CEA binding availability of the cell lines used in this study. Note the (arbitrary) classification in high $(>5 \%$ specific binding) and low ( $<5 \%$ specific binding) binding of DTPA-hMN14-IRDye700DX.

\section{Abbreviations}

BB: Binding buffer; CEA: Carcinoembryonic antigen;

DTPA: Diethylenetriaminepentaacetic acid; EDTA: Ethylenediaminetetraacetic acid; hMN-14: Labetuzumab; MES: M 2-(N-morpholino)ethanesulfonic acid; NIR: Near infrared; PBS: Phosphate-buffered saline; PDT: Photodynamic therapy; ROS: Reactive oxygen species; tPDT: Targeted photodynamic therapy

\section{Acknowledgements}

The authors thank the biotechnicians of the Preclinical Imaging Center (PRIME, Nijmegen, The Netherlands) for their technical assistance with the animal experiments.

\section{Authors' contributions}

FE and DB collected the data and contributed to early versions of the manuscript. FE analyzed, interpreted the data, and wrote the manuscript. MR and $\mathrm{OB}$ conceptualized research questions and the study setup and contributed to writing of the final manuscript. DG contributed to writing the final manuscript. All authors read and approved the final manuscript.

\section{Funding}

No specific funding was gained for the current research.

\section{Availability of data and materials}

The datasets used and/or analyzed during the current study are available from the corresponding author on reasonable request.

\section{Ethics approval and consent to participate}

All animal experiments were approved by the Institutional Animal Welfare Committee of the Radboud University Medical Center and were conducted in accordance to the guidelines of the Revised Dutch Act on Animal Experimentation (2014).

Consent for publication

Not applicable 


\section{Competing interests}

DG is a retired Founder and Chairman (emeritus) of Immunomedics, Inc., and IBC Pharmaceuticals, Inc. The other authors declare no other competing interests.

\section{Author details}

'Department of Radiology and Nuclear Medicine, Radboud University Medical Center, PO Box 9101, 6500 HB Nijmegen, The Netherlands. ${ }^{2}$ Center for Molecular Medicine and Immunology, Mendham, NJ, USA.

${ }^{3}$ Immunomedics, Inc. and IBC Pharmaceuticals, Inc., Morris Plains, NJ, USA.

Received: 5 September 2019 Accepted: 28 November 2019 Published online: 11 December 2019

\section{References}

1. Amri R, Bordeianou LG, Sylla P, Berger DL. Association of radial margin positivity with colon cancer. JAMA Surg. 2015;150:890-8. https://doi.org/10. 1001/jamasurg.2015.1525.

2. Bhangu A, Ali SM, Darzi A, Brown G, Tekkis P. Meta-analysis of survival based on resection margin status following surgery for recurrent rectal cancer. Color Dis. 2012;14:1457-66. https://doi.org/10.1111/j.1463-1318.2012.03005.x.

3. Petrelli F, Zaniboni A, Ghidini A, Ghidini M, Turati L, Pizzo C, et al. Timing of adjuvant chemotherapy and survival in colorectal, gastric, and pancreatic cancer. A Systematic Review and Meta-Analysis. Cancers (Basel). 2019;11. doi:10.3390/cancers11040550.

4. Alberti P. Platinum-drugs induced peripheral neurotoxicity: clinical course and preclinical evidence. Expert Opin Drug Metab Toxicol. 2019. https://doi. org/10.1080/17425255.2019.1622679.

5. Tampa M, Sarbu MI, Matei C, Mitran Cl, Mitran Ml, Caruntu C, et al. Photodynamic therapy: a hot topic in dermato-oncology. Oncol Lett. 2019; 17:4085-93. https://doi.org/10.3892/ol.2019.9939.

6. Civantos FJ, Karakullukcu B, Biel M, Silver CE, Rinaldo A, Saba NF, et al. A review of photodynamic therapy for neoplasms of the head and neck. Adv Ther. 2018;35:324-40. https://doi.org/10.1007/s12325-018-0659-3.

7. Mahmoudi K, Garvey KL, Bouras A, Cramer G, Stepp H, Jesu Raj JG, et al. 5aminolevulinic acid photodynamic therapy for the treatment of high-grade gliomas. J Neuro-Oncol. 2019;141:595-607. https://doi.org/10.1007/s11060019-03103-4.

8. Inoue K. 5-Aminolevulinic acid-mediated photodynamic therapy for bladder cancer. Int J Urol. 2017;24:97-101. https://doi.org/10.1111/iju.13291.

9. Moore LS, de Boer E, Warram JM, Tucker MD, Carroll WR, Korb ML, et al. Photoimmunotherapy of residual disease after incomplete surgical resection in head and neck cancer models. Cancer Med. 2016;5:1526-34. https://doi. org/10.1002/cam4.752.

10. Castano AP, Mroz P, Hamblin MR. Photodynamic therapy and anti-tumour immunity. Nat Rev Cancer. 2006;6:535-45. https://doi.org/10.1038/nrc1894.

11. Mroz P, Yaroslavsky A, Kharkwal GB, Hamblin MR. Cell death pathways in photodynamic therapy of cancer. Cancers (Basel). 2011;3:2516-39. https:// doi.org/10.3390/cancers3022516.

12. Sato K, Ando K, Okuyama S, Moriguchi S, Ogura T, Totoki S, et al. Photoinduced ligand release from a silicon phthalocyanine dye conjugated with monoclonal antibodies: a mechanism of cancer cell cytotoxicity after near-infrared photoimmunotherapy. ACS Cent Sci. 2018;4:1559-69. https:// doi.org/10.1021/acscentsci.8b00565.

13. Mitsunaga M, Ogawa M, Kosaka N, Rosenblum LT, Choyke PL, Kobayashi H. Cancer cell-selective in vivo near infrared photoimmunotherapy targeting specific membrane molecules. Nat Med. 2011;17:1685-91. https://doi.org/10. 1038/nm.2554.

14. Sharkey RM, Juweid M, Shevitz J, Behr T, Dunn R, Swayne LC, et al. Evaluation of a complementarity-determining region-grafted (humanized) anti-carcinoembryonic antigen monoclonal antibody in preclinical and clinical studies. Cancer Res. 1995;55:5935s-45s.

15. de Boer E, Warram JM, Hartmans E, Bremer PJ, Bijl B, Crane LM, et al. A standardized light-emitting diode device for photoimmunotherapy. Journal of nuclear medicine : official publication, Society of Nuclear Medicine. 2014; 55:1893-8. https://doi.org/10.2967/jnumed.114.142299.

16. Dobrucki J, Bleehen NM. Cell-cell contact affects cellular sensitivity to hyperthermia. Br J Cancer. 1985;52:849-55.

17. Leith JT, Heyman P, DeWyngaert JK, Dexter DL, Calabresi P, Glicksman AS. Thermal survival characteristics of cell subpopulations isolated from a heterogeneous human colon tumor. Cancer Res. 1983;43:3240-6.
18. Boswell CA, Tesar DB, Mukhyala K, Theil FP, Fielder PJ, Khawli LA. Effects of charge on antibody tissue distribution and pharmacokinetics. Bioconjug Chem. 2010;21:2153-63. https://doi.org/10.1021/bc100261d.

19. Rijpkema M, Bos DL, Cornelissen AS, Franssen GM, Goldenberg DM, Oyen WJ, et al. Optimization of dual-labeled antibodies for targeted intraoperative imaging of tumors. Mol Imaging. 2015;14. https://doi.org/ 10.2310/7290.2015.00015.

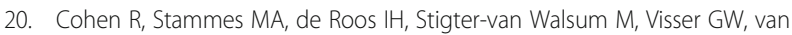
Dongen GA. Inert coupling of IRDye800CW to monoclonal antibodies for clinical optical imaging of tumor targets. EJNMMI Res. 2011;1:31. https://doi. org/10.1186/2191-219X-1-31.

21. Spring $B Q$, Rizvi I, Xu N, Hasan T. The role of photodynamic therapy in overcoming cancer drug resistance. Photochem Photobiol Sci. 2015;14: 1476-91. https://doi.org/10.1039/c4pp00495g.

22. Gonzalez-Exposito R, Semiannikova M, Griffiths B, Khan K, Barber L, Woolston A, et al. CEA expression heterogeneity and plasticity confer resistance to the CEA-targeting bispecific immunotherapy antibody cibisatamab (CEA-TCB) in patient-derived colorectal cancer organoids. J Immunother Cancer. 2019;7:101. https://doi.org/10.1186/s40425-019-0575-3.

23. Yan C, Hu Y, Zhang B, Mu L, Huang K, Zhao H, et al. The CEA-/lo colorectal cancer cell population harbors cancer stem cells and metastatic cells. Oncotarget. 2016;7:80700-15. https://doi.org/10.18632/oncotarget.13029.

24. Nagaya T, Friedman J, Maruoka Y, Ogata F, Okuyama S, Clavijo PE, et al. Host immunity following near-infrared photoimmunotherapy is enhanced with PD-1 checkpoint blockade to eradicate established antigenic tumors. Cancer immunology research. 2019;7:401-13. https://doi.org/10.1158/2326-6066.Cir-18-0546.

25. Mitsunaga M, Nakajima T, Sano K, Choyke PL, Kobayashi H. Near-infrared theranostic photoimmunotherapy (PIT): repeated exposure of light enhances the effect of immunoconjugate. Bioconjug Chem. 2012;23:604-9. https://doi.org/10.1021/bc200648m.

26. Lütje S, Heskamp S, Franssen GM, Frielink C, Kip A, Hekman M, et al. evelopment and characterization of a theranostic multimodal anti-PSMA targeting agent for imaging, surgical guidance, and targeted photodynamic therapy of PSMA-expressing tumors. Theranostics. 2019;9(10):2924-38. https://doi.org/10.7150/thno.35274.

27. Wei W, Jiang D, Ehlerding EB, Barnhart TE, Yang Y, Engle JW, et al. CD146targeted multimodal image-guided photoimmunotherapy of melanoma. Advanced science (Weinheim, Baden-Wurttemberg, Germany). 2019;6: 1801237. doi:10.1002/advs.201801237.

28. Hammarstrom S. The carcinoembryonic antigen (CEA) family: structures, suggested functions and expression in normal and malignant tissues. Semin Cancer Biol. 1999;9:67-81. https://doi.org/10.1006/scbi.1998.0119.

29. Rijpkema M, Oyen WJ, Bos D, Franssen GM, Goldenberg DM, Boerman OC. SPECT- and fluorescence image-guided surgery using a dual-labeled carcinoembryonic antigen-targeting antibody. J Nucl Med. 2014;55:1519-24. https://doi.org/10.2967/jnumed.114.142141.

30. Liersch T, Meller J, Kulle B, Behr TM, Markus P, Langer C, et al. Phase II trial of carcinoembryonic antigen radioimmunotherapy with 1311-labetuzumab after salvage resection of colorectal metastases in the liver: five-year safety and efficacy results. Journal of clinical oncology : official journal of the American Society of Clinical Oncology. 2005;23:6763-70. https://doi.org/10. 1200/jco.2005.18.622.

31. Sharkey RM, Govindan SV, Cardillo TM, Donnell J, Xia J, Rossi EA, et al. Selective and concentrated accretion of SN-38 with a CEACAM5-targeting antibody-drug conjugate (ADC), labetuzumab govitecan (IMMU-130). Mol Cancer Ther. 2018;17:196-203. https://doi.org/10.1158/1535-7163.Mct-17-0442.

32. Hajjar G, Sharkey RM, Burton J, Zhang CH, Yeldell D, Matthies A, et al. Phase I radioimmunotherapy trial with iodine-131--labeled humanized MN-14 anticarcinoembryonic antigen monoclonal antibody in patients with metastatic gastrointestinal and colorectal cancer. Clin Colorectal Cancer. 2002;2:31-42. https://doi.org/10.3816/CCC.2002.n.009.

33. Koppe MJ, Soede AC, Pels W, Oyen WJ, Goldenberg DM, Bleichrodt RP, et al. Experimental radioimmunotherapy of small peritoneal metastases of colorectal origin. International journal of cancer Journal international du cancer. 2003;106:965-72. https://doi.org/10.1002/ijc.11304.

34. Dotan E, Cohen SJ, Starodub AN, Lieu CH, Messersmith WA, Simpson PS, et al. Phase I/II trial of labetuzumab govitecan (anti-CEACAM5/SN-38 antibody-drug conjugate) in patients with refractory or relapsing metastatic colorectal cancer. Journal of clinical oncology : official journal of the American Society of Clinical Oncology. 2017;35:3338-46. https://doi.org/10. 1200/jco.2017.73.9011. 
35. Dong W, Shi J, Yuan T, Qi B, Yu J, Dai J, et al. Antibody-drug conjugates of 7-ethyl-10-hydroxycamptothecin: sacituzumab govitecan and labetuzumab govitecan. Eur J Med Chem. 2019;167:583-93. https://doi.org/10.1016/j. ejmech.2019.02.017.

36. Shirasu N, Yamada H, Shibaguchi H, Kuroki M, Kuroki M. Potent and specific antitumor effect of CEA-targeted photoimmunotherapy. International journal of cancer Journal international du cancer. 2014;135:2697-710. https://doi.org/10.1002/ijc.28907.

37. Hekman MC, Rijpkema M, Muselaers CH, Oosterwijk E, Hulsbergen-Van de Kaa CA, Boerman OC, et al. Tumor-targeted dual-modality imaging to improve intraoperative visualization of clear cell renal cell carcinoma: a first in man study. Theranostics. 2018;8:2161-70. https://doi.org/10.7150/thno. 23335.

\section{Publisher's Note}

Springer Nature remains neutral with regard to jurisdictional claims in published maps and institutional affiliations.

\section{Submit your manuscript to a SpringerOpen ${ }^{\circ}$ journal and benefit from:}

- Convenient online submission

- Rigorous peer review

- Open access: articles freely available online

High visibility within the field

- Retaining the copyright to your article

Submit your next manuscript at $\boldsymbol{\nabla}$ springeropen.com 